\title{
Reknit Revolution: knitwear design for the domestic circular economy
}

\author{
Amy Twigger Holroyd \\ Nottingham Trent University \\ School of Art \& Design, 50 Shakespeare Street, Nottingham NG1 4FQ, UK \\ $+44(0) 1158488249$ \\ amy-twigger.holroyd@ntu.ac.uk
}

Amy Twigger Holroyd is a designer, maker and researcher who has been active in the emerging field of fashion and sustainability since 2004. Her designs have been featured in many books, publications and exhibitions, from Vogue to Fashion Theory. She is a Senior Lecturer in the School of Art \& Design at Nottingham Trent University. 


\title{
Reknit Revolution: knitwear design for the domestic circular economy
}

\begin{abstract}
An ongoing initiative seeks to encourage hand knitters to expand their practices to encompass reknitting: the reworking of existing knitted items using knit-based skills, techniques and knowledge. Reknitting offers potential benefits in terms of promoting repair and re-use by individual users within the domestic sphere, thereby contributing to the "domestic circular economy". Although reknitting was a common element of hand knitting practice in the past, the knowledge of how to open, alter, unravel and reknit has largely been lost. This gap in practice presents a fertile challenge for contemporary knitted textile design.

A first phase of activity saw the initial development and testing of reknitting techniques suitable for the garments in our wardrobes today. This project demonstrated that it is possible to engage knitters with reknitting, but that support is needed to overcome barriers to participation and shift perceptions of what is both possible and desirable. Three interconnected elements of support are discussed: inspiration, information and confirmation.
\end{abstract}

A second phase of activity, centered around an exhibition in a public gallery, aimed to communicate the techniques to a wider knitting community. Exhibits included physical garments, a large-scale infographic and a film; adaptable instructions were developed and published on a companion website. Through analysis of these exhibits and instructions, categories within each of the three elements of support were identified to create a more detailed taxonomy of support for domestic reknitting practice.

Reflection on the progress of this initiative indicates the direction for future activity: raising broader awareness of reknitting, primarily via online channels, and structuring opportunities for participation to build a mutually supportive community of practice. It is anticipated that the taxonomy of support could be applied to other initiatives seeking to promote domestic circular economy activity.

\section{Keywords}

knitting; repair; mending; amateur; sustainability; circular economy 


\section{Introduction}

When is a garment finished? We tend to think that when the last seam is sewn, the making process is complete. Our clothes are worn, stored and discarded, but rarely transformed. But if this norm is challenged, new opportunities for both creativity and sustainability open up.

This article discusses an ongoing initiative that seeks to encourage hand knitters to expand their craft practices to encompass reknitting: the reworking of existing knitted items using knit-based skills, techniques and knowledge. Reknitting offers potential benefits in terms of promoting repair and re-use by individual users within the domestic sphere, thereby contributing to the constellation of initiatives that, together, will build a circular economy.

Reknitting was a common element of domestic knitting practice in the past, when necessity impelled people to exploit the inherent "tinkerability" of the weft knitted structure. When items became worn at the cuffs, elbows or collars those elements would be unravelled and replaced (Pearson 1980; Sundbø 2000). During World War II rationing prompted an intensification of reknitting activity, with instructions providing guidance in how to reclaim yarn from worn garments and combine these precious materials into fresh items (Koster and Murray 1943). Since this period, as knitting has shifted from a domestic necessity to an optional leisure activity, reknitting activity has dwindled. The knowledge of how to open, alter, unravel and reknit has largely been lost. This gap in practice presents a fertile challenge for contemporary knitted textile design.

The reknitting techniques under discussion were initially developed and creatively explored during a doctoral research project (Twigger Holroyd 2013), described here as Phase 1. Although the primary aim of this research, which involved collaboration with a group of six amateur knitters, was the generation of new knowledge about the lived experience of making and remaking, the outputs of the design activity - the reknitting techniques themselves were also felt to have value. The project demonstrated that it is possible to engage knitters with reknitting, but that support is needed to help makers shift their perceptions of what is both possible and desirable.

During a further phase of activity, greater emphasis was placed on the communication of the techniques to a wider knitting community. The centrepiece of Phase 2 was Units of Possibility: The Reknit Revolution, an exhibition in Summer 2017 at Rugby Art Gallery \& Museum (UK). The exhibition subsequently toured from this public gallery to two iterations of a major enthusiast event, The Knitting \& Stitching Show. Across all three installations, the exhibition attracted over 15,000 visitors. It incorporated several exhibits intended to inform and inspire visitors about the creative possibilities of reknitting, including physical garments, a large-scale infographic and a film. A series of workshops conducted in the months before 
the exhibition generated eleven reknitting projects created by six participants, which were also exhibited. An accompanying website, reknitrevolution.org, acts as an openly accessible repository of instructional materials relating to the project.

This article will first explore what I term the "domestic circular economy": the repair and reworking of items by users within the domestic context. It will then discuss Phase 1 of the reknitting project, considering barriers to participation and the ways in which these barriers were overcome in this small-scale pilot initiative. Three elements of support - inspiration, information and confirmation - will be considered. Moving on, it will analyse the various activities that took place in Phase 2, drawing on personal reflections and visitor responses to consider the degree to which the exhibition enabled the scaling up of support for reknitting. Categories within each of the three elements will be identified, creating a more detailed taxonomy of support for domestic reknitting practice. Finally, the article will look forward to a third phase of activity, exploring the potential for further development.

\section{The domestic circular economy}

The circular economy is perhaps best introduced using the "cradle to cradle" concept famously proposed by McDonough and Braungart (2002). As an alternative to the linear industrial system of "take, make and dispose", cradle to cradle thinking views materials as nutrients that can flow through the system time after time. Unlike the vast majority of current recycling initiatives, in which materials decline in quality as they are reclaimed and transformed, true circularity demands that quality is maintained indefinitely.

The holy grail of sustainable design is the development of products whose materials can be eternally re-used. When they reach the end of their useful lives, they could be taken back to their base materials and transformed into a completely different form or function. This would be, in short, a product lifecycle that behaves just like a natural one, repeatedly transforming materials for new cycles of growth (Goldsworthy 2014: 250).

The circular economy is built on this idea, creating "a continuous positive development cycle that preserves and enhances natural capital, optimises resource yields, and minimises system risks by managing finite stocks and renewable flows" (Ellen MacArthur Foundation 2015). The cyclical approach is not confined to the re-use of base materials; recycling of this type should arguably be seen as a last resort once less energy-intensive opportunities for reuse have been exhausted. The circular economy system diagram created by The Ellen MacArthur Foundation (2015) illustrates four nested levels of re-use, descending in terms of scale and involving different stakeholders: 
- recycle to reclaim base materials (parts manufacturers)

- refurbish and remanufacture (product manufacturers)

- facilitate re-use and redistribution (service providers)

- maintain and prolong useful life (individual users)

Goldsworthy (2014) distinguishes between re-active approaches to re-use and recycling, which respond to waste generated by the present linear system, and pro-active approaches, in which the reclamation of high quality base materials is considered from the earliest stages of design. She argues that both approaches are necessary in order to deal with the materials in circulation today while starting to develop a more robust circular system. Thus, overall the circular economy requires us to "keep resources in use for as long as possible, extract the maximum value from them whilst in use, then recover and regenerate products and materials at the end of each service life" (WRAP 2016).

The circular economy concept has gained traction among businesses and policy-makers in recent years, and work is progressing across a range of industrial sectors to investigate the development of this alternative system. As Perella (2014) explains, "Ground-level innovation in this field is being driven by large corporations who are piloting business models based on leasing, product performance, remanufacture, and extended lifecycle thinking." These corporations are motivated by long-term business benefits such as protection from fluctuating commodity prices (Hower 2016).

While this work has obvious value, it is crucial that we do not fall into the trap of associating the circular economy exclusively with commercial activities and thereby sideline the domestic arena. Action by individuals has great potential in terms of clothing, because textiles are accessible materials with an inherent capacity for repair. As Fletcher (2016) points out, such actions require little in the way of energy or material inputs. Although individual instances of repair and remaking may seem insignificant, when aggregated they have the potential to deliver genuine change. And while repair and remaking activities at this scale are, by their nature, re-active, they still have an important part to play in extending product life and contributing to a circular economy.

In order to focus attention on the domestic sphere, I propose that we should consider the notion of the "domestic circular economy". Inspired by the diverse economies framework put forward by Gibson-Graham et al. (2013) and the social economy discussed by Murray (2012), this encompasses all activities contributing to the circular economy at the domestic level, including all of the unpaid, non-market, reciprocal and gift-based processes involved in dayto-day household life. While activity in this sphere is of little interest to large corporations, there is a potent opportunity for design activists, such as myself, to make a contribution. 
Research indicates that very little activity is currently taking place in the domestic circular economy. For most people mending, if practised at all, is limited to small tasks such as replacing buttons and sewing up hems (Fisher et al. 2008). The public-facing Love Your Clothes campaign, associated with the UK's Sustainable Clothing Action Plan, is seeking to address this lack of knowledge by providing information on repair and refashioning. However, as Goldsworthy (2014: 252) observes, "changes in consumer habits are very difficult to achieve". Furthermore, any attempt to encourage people to make and repair their garments must contend with the mixed meanings of homemade and mended clothes in contemporary culture (Twigger Holroyd 2017). In short: even if garments are repaired, it is far from certain that they will return to regular wear.

It may be more productive to target the growing numbers of people who enjoy making clothes as a leisure activity, inviting them to consider using their skills to rework, rather than always to make new. This is already taking place in terms of sewn garments: refashioning is seeing a notable resurgence, with support available via books, workshops and online resources. The reknitting initiative under discussion seeks to encourage experienced knitters to extend their practices in a similar way.

\section{Phase 1}

As outlined in the introduction, in the past reknitting would have been an integral part of much domestic knitting practice. Worn elements of garments and accessories could be removed and replaced; alternatively, entire items could be unravelled in order to reclaim the yarn. These commonplace practices fell out of use as readymade items became cheaper and repair consequently became the exception, rather than the norm. At the same time knitting was transitioning into a hobby, undertaken out of choice rather than necessity, and an activity centred upon the creation of new items.

The Phase 1 project sought to address the lack of reknitting activity in contemporary knitting practice by developing reknitting techniques appropriate to the items in our wardrobes today. Although these garments utilise the same basic weft-knitted structure as the hand-knitted pieces that would typically have been reworked in the past, their specific characteristics often differ. For example, many of today's items are made from fine-gauge knitted fabrics, with stitches much smaller than would normally be hand knitted; this disguises the structure's inherent capacity for alteration and poses additional challenges for the aspiring reknitter. In the early stages of the research I accessed a range of published instructions for reknitting, with the earliest advising how "to freshen a last season's jersey" with a new collar and cuffs (Klickmann 1916: 18). A wealth of publications from the era of rationing in Britain (e.g. Koster 
and Murray 1943) included instructions for procedures including refooting a pair of socks and straightening yarn reclaimed from unravelled items. In more recent publications, guidance on reknitting aimed to help knitters adapt newly-knitted items that had turned out too large or too small. To complement these sources of guidance, I used my knowledge as a designer and maker of knitwear to generate new ideas for reknitting. For example, I had already begun to develop a technique that I term "stitch-hacking". This is the reconfiguration of stitches in an existing knitted fabric, achieved by laddering and reforming columns of loops. Stitch-hacking uses an established repair technique in a new context: to change a fabric to a new design rather than repairing it back to its former structure (Figure 1).

Through multiple stages of iterative development I combined these diverse ideas together to create a "spectrum" of reknitting techniques (Figure 2). The reknit spectrum captures the full range of technical processes, or "treatments", that could be used to alter and rework an item of knitwear. Each treatment is endlessly variable, considering the specifics of the original garment and factors such as the colour, scale and gauge of the alteration. The treatments are grouped according to the way in which the original garment is "opened", rather than - as is usually the case with historical instructions - the problem to be addressed. The steps involved in each treatment are identified, indicating in general terms the process by which it would be achieved. This approach was adopted in order to highlight the range of options available and to invite knitters to consider diverse futures for their garments.

In a series of four day-long workshops I worked with a group of six amateur knitters to test the spectrum and the reknitting treatments. The project culminated in each participant reworking an item from their own wardrobe; a range of treatments were employed, including cut open and trim (Figure 3), replace edge section (Figure 4) and separate element (Figure 5). The techniques were used to address problematic items: garments that were significant enough to be kept, but with issues that excluded them from wear. When reflecting on the project, the participants expressed satisfaction with the completed projects and a fascination with the reknitting process. They responded positively to the challenge of reknitting, finding that the experience offered the many benefits that they associated with their usual knitting practices. Analysis of the project activities indicated that the knitters were drawing on their tacit making knowledge - gained from their experience of "conventional" knitting - when engaging in the unfamiliar reknitting processes. Thus, their prior practices had equipped them with the practical and creative skills required for reknitting, which the structure of the project enabled them to "unlock".

Despite the success of these individual projects, the Phase 1 research also highlighted barriers to engagement in reknitting practice, indicating why - given the interest in restyling sewn garments - there is not already a whirlwind of reknitting activity taking place in the 
domestic sphere. First, and most fundamentally, the idea of reworking a knitted item using knitting skills and knowledge is simply not in circulation. There is a widespread perception of knitting as a process of construction, rather than reconstruction. This perception is shaped by the established understanding of what it is to "do" knitting: use a set pattern, prescribed yarn and needles to construct a finished item. In order for a culture of reknitting to develop, this understanding needs to be challenged. Furthermore, repair must be reframed as a positive, creative act rather than a practice associated with poverty.

Even when knitters become aware of the idea of reknitting - as in this project - a range of issues arise. These issues shape both the experiences of those who might want to reknit, and the efforts of those who aspire to support them. Concerns include:

- deconstruction: the idea that if you "open" a knitted fabric, it will disintegrate uncontrollably

- lack of skill: knitters frequently fail to recognise their transferable tacit knowledge

- differences in gauge: many pre-existing garments are constructed from smaller stitches than hand-knitted fabrics

- unconventional "pattern": because garments to be reknitted are endlessly variable, any instructions must be open-ended

- need to design: open-ended instructions require the knitter to make creative decisions, a task that can feel daunting to amateur makers

- aesthetic appeal: anxiety about whether the reworked item will look intentional and "whole"

- contingency: a recognition that unexpected problems often emerge during repair

The Phase 1 project demonstrated that it is quite possible to shift knitters' thinking around all of these potential barriers. With support, the participants embraced the concept of reknitting and began to see it as part of their normal knitting practice. By playing around with scrap garments, they gained a deeper understanding of the knitted structure and its capacity for deconstruction. They came to recognise their own tacit making knowledge and draw on their experience to use open-ended instructions. They gained confidence in their ability to design for themselves and developed strategies for ensuring that their items looked coherent and finished. They even came to see reworking as a rewarding journey into the unknown. An alternative way of thinking about barriers is to consider the steps that would be involved if a novice reknitter were to successfully execute an alteration. These steps are summarised in Figure 6.

Any initiative aiming to support knitters to engage in reknitting must address the barriers discussed and also provide assistance at each step of the reknitting journey. The Phase 1 
project demonstrated that multifaceted support is required. This support must not only offer practical advice on how to reknit; it must also achieve the more nebulous goal of shifting perceptions of what is possible and desirable in terms of domestic knitting and repair practices. Reflecting on the project, I identified three interconnected elements within this support, all of which proved to be crucial: inspiration, information and confirmation. In order to foster a culture of reknitting, all three elements would have to be created on a larger scale.

The first element, inspiration, aims to provide positive examples of reknitting that will encourage knitters to engage in the activity themselves. In the Phase 1 project I found that sharing precedents - whether stories of reknitting from the past or sample garments that I had produced (such as the sampler shown in Figure 7) - helped the participants to develop a mental space for this approach and become enthusiastic about the possibilities.

Conversations from the workshops revealed that several of the knitters were inspired by the notion of connecting with a practice from the past. The examples helped them to grasp the diversity of what reknitted garments could look like, and begin to generate their own ideas.

The second element, information, encompasses all of the advice needed to assist knitters in undertaking reknitting projects. As described above, the design of this information presents a challenge: because every item to be reknitted will be different, the instructions must be open enough for the knitter to adapt but detailed enough to be of use during a complex process. For the Phase 1 project I developed a range of materials, including general advice on how to approach a reknitting project; information on each treatment including step-by-step visual instructions, stitch patterns and specialised advice; instructions on how to carry out operations common to many of the treatments; and tips and exercises to develop design skills. The primary aim underpinning these materials was to provide support while I worked with the participants of the Phase 1 project. They were not intended to support independent activity and thus it was evident that further development would be necessary in order to maximise their usefulness; this was the focus for the instructions developed in Phase 2. The final element needed to support reknitting is confirmation. This relates to confidence: makers generally need encouragement and positive feedback from their peers in order to complete their projects, feel happy with the outcomes and gain reassurance that reknitting is a worthwhile activity. The Phase 1 project demonstrated the importance of this type of support, with one of the knitters commenting: "I need to feed off other people, I think, to get ideas, and then to gain confidence in my ideas." Homemade clothes are marginal in contemporary culture; it is rather risky to make clothes without the sanctioning influence of professional manufacture or even a professionally-designed knitting pattern for support. By making alongside other people, the knitters benefited from an alternative source of sanctioning. 


\section{Phase 2}

The Phase 2 activity was prompted by the invitation to create a solo exhibition focusing on the reknitting techniques at a public art gallery in Summer 2017. This activity provided a valuable opportunity to test my ideas about support for reknitting through engagement with a much larger group of potential reknitters. As will be explained, I found that I was able to identify different categories of inspiration, information and confirmation, developing a more detailed taxonomy of support.

Around eight months before the exhibition opened I ran a series of three participatory workshops, held at the gallery and promoted to knitters in the local area. These workshops provided the opportunity to guide a group of participants through some introductory reknitting techniques and, in the process, test instructional materials. While 14 people attended the workshops overall, a core group of six participants engaged fully with the project and chose to develop reknitting projects for the exhibition. Each participant completed between one and three projects, all of which were exhibited (Figure 8) along with explanatory descriptions, written by the makers themselves. I included these projects in the exhibition in order to provide inspiration to visitors and show the accessibility and adaptability of reknitting. I felt that community-generated projects such as these would help knitters to see that their peers had successfully engaged in this alternative making activity and thus more readily recognise their own transferable tacit knowledge.

Inspiration was also the focus of three further exhibits: a reworked fisherman's gansey from the 1930s loaned by the Scottish Fisheries Museum and two series of my own work. The Stitch-hack series (including the item shown in Figure 9) comprises five found garments, into which I have retrospectively configured new designs that explore questions of ownership and authorship. The five reknitted cast-offs in the Cathedral Cardigan series (Figure 10) interpret the construction details of gothic cathedrals in diverse ways. I created all of these intricate "showcase" pieces with the express intention of intriguing the viewer, aiming to draw them in to consider the processes used and provide inspirational examples of how reknitting treatments can be creatively applied to diverse garments. Thus, within these first three components of the exhibition I can identify three categories of inspiration: communitygenerated, historical and showcase.

The centrepiece of the Units of Possibility exhibition was an updated version of the reknit spectrum initially created during Phase 1, presented as a five metre-wide infographic and illustrated with half-scale garments and images of the treatments in progress (Figure 11). There are some significant differences between this spectrum and the previous version. First, 
I removed the graphic elements that indicated the steps involved in working each treatment; I felt that this level of detail distracted from effective communication of the reknitting principles. Second, the line-up of treatments was altered. Some treatments were grouped and/or renamed; for example, cut open and trim became cardiganise - a more memorable and evocative term. One new treatment, reinvent, was added in recognition of unanticipated approaches developed by the participants during the Phase 1 and Phase 2 workshops.

The half-scale sample garments were created as a batch of basic ecru crew-neck jumpers, using industrial machinery and construction techniques. The reknitting treatments were worked using bright red yarn of a standard hand-knit weight. This aesthetic consistency was selected in order to communicate the principle of each technique as straightforwardly as possible. As I was aiming to showcase the general idea of the treatment, I wanted to create a design that was relatively simple; this was a challenge because some of the treatments, such as embellish, are inherently decorative and cannot be carried out in a "neutral" way.

Feedback from visitors to The Knitting \& Stitching Show installations, which I documented while invigilating the exhibition, indicated that the spectrum provided support in the form of information - albeit at an overview level, providing guidance on the overall concept of each treatment, rather than the detail needed to act. I would, therefore, categorise this as general information. Comments provide evidence that the presentation of the infographic was effective in communicating these principles:

Your presentation's really wonderful. It's really instant.

Great concept - very well described.

As a dyslexic, I can't follow "recipes" - patterns - they make me demoralised and I give up. But I understood this immediately.

Furthermore, feedback indicated that the array of options displayed on the spectrum provided inspiration. I would place this inspiration into a different category to the previous cases, which I term principle-focused. While I feel that all of the categories of inspiration have value, visitor comments suggested that the principle-focused inspiration provided by the spectrum was the most effective in providing a pathway to action:

So clever ... gives you lots of ideas and inspiration.

I'd just been saying that I didn't know what to do with worn out knits other than stick them in the washing machine and try to felt them.

I'll be looking at my wardrobe in a whole new light now.

Returning to the information element of support, two further aspects of the exhibition provided information that would fall into a second category: technique-specific. These were a 
film, created in collaboration with filmmaker Jonathan Hamilton, and online instructions published on the reknitrevolution.org website. The film documented the process of creating three of the half-scale garments displayed within the spectrum: stitch-hack, replace cuff and cardiganise. It was not intended as instructional material, but rather a means of showcasing the skills involved in reknitting in an engaging manner. Yet it played a valuable role in the exhibition, demonstrating to intrigued visitors how each of the three transformations had taken place.

The online instructions were developed with the aim of creating standalone guidance that could be accessed independently by knitters interested in engaging in reknitting for the first time. As I have explained, the process of reknitting cannot be guided using a conventional pattern. The use of open-ended instructions poses a potential challenge for knitters used to a more prescriptive format, just as the creation of such guidance poses a challenge for designers. Therefore, I aimed to make the instructions as user-friendly as possible. Building on the materials I had created for the reknitting resource during Phase 1, I developed a standard format that included images of the sample garment in process and at completion (such as those shown in Figure 12), an outline of the principles of the treatment and some "top tips". These tips include the recommendation to try out each of the techniques on some scrap fabric before embarking on the project, as the workshops during Phases 1 and 2 indicated that playing with deconstruction techniques had helped knitters to overcome their fear of cutting or opening a knitted fabric.

The instructions also cover a series of questions, namely: What does it involve? What could it look like? How do I do it? How do I design it? The How do I do it? sections each comprise three videos, recorded using a table-mounted camera to focus on my hands as I worked and explained the relevant techniques. The How do I design it? sections utilise diagrams and charts to visually communicate the calculations needed for each treatment. For example, the fine gauge of much of the knitwear in our wardrobes today - a potential barrier to reknitting means that the replace cuff treatment often requires a change of gauge, from a large number of small stitches to a smaller number of larger stitches. This procedure requires mathematical calculations relating to the ratio of old to new stitches, and consideration of the aesthetic of different ratio options. Although in Phase $1 \mathrm{I}$ had created a number of tools to guide this deceptively complex process, they were rather difficult to follow. For the new instructions I created simpler tools including a chart which groups fabric weights and visually displays the ratio relationships between them (Figure 13).

For the Knitting \& Stitching Shows, I created concise print versions of each of the three sets of instructions, which signposted knitters to the online resources. Over 3,300 sets of instructions were distributed. As I estimated that visitors typically each took two sets, this 
represents the active engagement of around $22 \%$ of visitors. I felt that this was a positive indication of the level of interest garnered by the exhibition and the appetite for treatmentspecific information. Visitor comments provided evidence of an intention to use the instructions for particular projects:

Cardiganise - great! I have all these boring jumpers, now I can do something with them rather than give them to the charity shop.

There's definitely at least one thing at home that'll benefit from this.

The third and final element of support identified in Phase 1, confirmation, relates to the confidence that makers gain from interacting with their peers while engaging in reknitting. I see this type of support as being particularly important in overcoming potential barriers to reknitting, including the need for knitters to design for themselves and the concern over whether a garment will look "whole" and "intentional" after reknitting alterations. Interaction with others certainly helps reknitters to overcome the hurdles inevitably thrown up by the contingency of repair. More broadly, confirmation helps to frame reknitting as a worthwhile and socially acceptable activity - and reknitted garments as valid and socially acceptable items. In Phase 1, confirmation was generated within the group of six participants. The same thing occurred during the Phase 2 workshops; the knitters gave each other encouragement and constructive feedback.

While the exhibition did not provide opportunities for this interpersonal category of confirmation, on reflection I feel that it helped potential reknitters to gain confidence in a different way. I would term this category validating confirmation. For example, the showcasing of community-generated projects not only provides inspiration to visitors, but also subtly portrays reknitting in a positive light. In fact, the same could be said about the exhibition overall: by placing this work in an prestigious space and giving it a public platform, a message of value is constructed and communicated.

As I have explained, during the course of developing and reflecting on the various components of the Phase 2 exhibition, I was able to identify different categories within each of the three elements of support. The resulting taxonomy - which I intend to use in planning a further phase of reknitting promotion, to be outlined in the next section - is summarised in Figure 14.

\section{Looking forward}

The positive response garnered by the Phase 2 exhibition leads me to look forward to further reknitting promotion. But what should Phase 3 involve? The goal would be to make the 
reknitting materials even more widely accessible; although an exhibition has been shown to be a productive means of engaging visitors, its reach is naturally limited. Thus, my focus would be the use of online platforms to raise awareness. The first step would be to enhance the materials hosted on the reknitrevolution.org website. I intend to add further techniquespecific information, providing guidance for additional treatments. There is also a need to communicate the aspects of the exhibition that provided inspiration and general information on the website. These tasks are reasonably straightforward; I anticipate that it will be more difficult to find ways to build confirmation online.

The validating type of confirmation is the more accessible of the two categories; the prestige of the exhibition could be mirrored, to some extent, by the construction of a well-designed online home and supported by coverage in enthusiast magazines and blogs. Further exhibition opportunities will also be explored. Interpersonal confirmation is more complicated. This crucial support relates to the two-way conversations that give makers confidence in their reknitting projects. Existing knitting or mending groups could be a good source of interaction; in these contexts, participants typically take along work in progress or completed projects to share with others. Yet a lone reknitter may find themselves acting as a pioneer in these circumstances, raising awareness rather than necessarily gaining support and encouragement.

Interpersonal support could take place online, either in a dedicated group or by using a hashtag to connect relevant posts on social media platforms such as Instagram. This practice will be familiar to many knitters; as Bratich and Brush (2011:242) suggest, "the knitting circle now meshes with the World Wide Web". The use of a hashtag has worked well to promote the related concept of "visible mending", with over 13,000 posts on Instagram alone. In fact, based on this model I feel that the sharing of reknitting projects online would create a productive blend of all three elements of support.

As there is not yet a community of reknitting practitioners ready to post their projects and provide constructive feedback to one another, action must be taken to kickstart activity. I aim to do this by posting inspiration and information on social media to galvanise interest and subsequently organising coordinated "knitalongs": this is an established format in which participants work on similar projects simultaneously, providing mutual support and encouragement. For example, the first knitalong could invite knitters to have a go at cardiganising a jumper, using the technique-specific instructions already available on the website.

\section{Conclusion}


This article has explored an initiative to promote the use of knitting skills to rework items within the wardrobe and thereby contribute to what I have termed the "domestic circular economy". While non-market domestic activity is inevitably of little interest to commercial corporations, it provides an important opportunity to extend product life and delay the need for more energy-intensive processes of re-use, remanufacture and recycling.

With a recent resurgence of interest in making clothes at home, a significant community of knitters have the skills required to rework existing garments. A range of barriers currently discourage this community from contemplating reknitting projects, including a lack of awareness that it is possible to knit without "knitting new". As Tulloch (2017: 3) observes, the processes involved in reknitting "scare and inspire simultaneously". The development of resources to support knitters in overcoming their terror presents an unconventional, though immensely satisfying, challenge for knitted textile designers such as myself.

Analysis of the support provided in Phase 1 identified three interconnected elements: inspiration, information and confirmation. All three are needed to help knitters to embark upon and accomplish reknitting projects in the home, and will need to be constructed at a larger scale if reknitting is to gain in popularity. I attempted to do this in the exhibition created during Phase 2. In doing so, a taxonomy of support to identify different categories within each element was developed. I found that the exhibition was particularly effective in providing inspiration, information and a general type of confirmation. Reflection on the progress of this initiative to date has indicated the direction for Phase 3: raising broader awareness of reknitting, primarily via online channels. To build capacity for the elusive interpersonal category of confirmation, I plan to structure opportunities for knitters to engage in reknitting. The aim is that this practice will gradually start to take on a life of its own, create a community of enthusiasts, and - in the process - help to shift perceptions of both knitting and repair.

Stepping back from the case of reknitting, I would argue that the three elements of support and the categories within them - identified in this article are crucial to any initiative seeking to promote activity within the domestic circular economy. It is my hope that this discussion will be of use to others attempting to develop or evaluate such initiatives.

\section{Acknowledgements}

I would like to thank Nikki Grange and her team at Rugby Art Gallery \& Museum, Liz Cooper and The Knitting \& Stitching Show for their support in developing and touring the Units of Possibility exhibition. I would also like to thank Jonathan Hamilton for the creation of the Reknit Revolution film. 


\section{References}

Bratich, J. Z., and Brush, H. M. 2011. "Fabricating Activism: Craft-Work, Popular Culture, Gender," Utopian Studies, 22: 233-260.

Ellen Macarthur Foundation. 2015. "Circular Economy Overview." Available online at: https://www.ellenmacarthurfoundation.org/circular-economy/overview/concept (accessed March 3).

Fisher, T., Cooper, T., Woodward, S., Hiller, A. and Goworek, H. 2008. Public Understanding of Sustainable Clothing. London: Defra.

Fletcher, K. 2016. Craft of Use: Post-Growth Fashion. Abingdon: Routledge.

Gibson-Graham, J.K. 2013. Take Back the Economy: An Ethical Guide for Transforming Our Communities. Minneapolis: University of Minnesota Press.

Goldsworthy, K. 2014. "Design for Cyclability: Pro-Active Approaches for Maximising Material Recovery," Making Futures, 3: 250-261.

Hower, M. 2016. "8 Companies to Watch in the Circular Economy," GreenBiz, August 10. Available online at: https://www.greenbiz.com/article/8-companies-watch-circular-economy (accessed March 3).

Klickmann, F. 1916. Needlework Economies: A Book of Mending and Making with Oddments and Scraps. London: The Office of The Girl's Own Paper and Woman's Magazine.

Koster, J. and Murray, M. 1943. Knitted Garments for All. London: Odhams Press.

Mcdonough, W. and Braungart, M. 2002. Cradle-to-Cradle: Remaking the Way We Make Things. New York: North Point Press.

Murray, R. 2012. Global Civil Society and the Rise of the Civil Economy. In M. Kaldor, H.L. Moore and S. Selchow (eds.) Global Civil Society 2012: Ten Years of Critical Reflection. Basingstoke: Palgrave Macmillan: 144-165.

Pearson, M.R.R. 1980. Traditional Knitting of the British Isles: The Fisher Gansey Patterns of North East England. Newcastle: Esteem Press.

Perella, M. 2014. "Ten Things You Need to Know About the Circular Economy," Edie.net. Available online at: https://www.edie.net/news/5/10-things-you-need-to-know-about-thecircular-economy (accessed March 3).

Sundbø, A. 2000. Everyday Knitting: Treasures from a Ragpile. Norway: Torridal Tweed. 
Tulloch, C. 2018. "Units of Possibility: The Reknit Revolution," Fashion Practice, DOI: 10.1080/17569370.2017.1414508.

Twigger Holroyd, A. 2013. Folk Fashion: Amateur Re-Knitting as a Strategy for Sustainability. $\mathrm{PhD}$ thesis, Birmingham City University.

Twigger Holroyd, A. 2017. Folk Fashion: Understanding Homemade Clothes. London: I.B.Tauris.

WRAP. 2016. WRAP and the Circular Economy. Available online at: http://www.wrap.org.uk/about-us/about/wrap-and-circular-economy (accessed March 3).

\section{Figure captions}

Figure 1: The stitch-hacking technique.

Figure 2: The reknit spectrum (Phase 1 version)

Figure 3: Phase 1 reknitting project featuring cut open and trim treatment, created by Julia.

Figure 4: Phase 1 reknitting project featuring replace edge section treatment, created by Kiki.

Figure 5: Phase 1 reknitting project featuring separate element treatment, created by Alex.

Figure 6: Steps involved in a hypothetical first reknitting project.

Figure 7: Reknitting sampler.

Figure 8: Participant projects, installed in the Units of Possibility exhibition at Rugby Art Gallery \& Museum. Courtesy of Jamie Grey at Rugby Art Gallery \& Museum.

Figure 9: 14GB, one item from the Stitch-hack series.

Figure 10: Cathedral Cardigan series, installed in the Units of Possibility exhibition at Rugby Art Gallery \& Museum. Courtesy of Jamie Grey at Rugby Art Gallery \& Museum.

Figure 11: The reknit spectrum (Phase 2 version), installed in the Units of Possibility exhibition at Rugby Art Gallery \& Museum. Courtesy of Jamie Grey at Rugby Art Gallery \& Museum.

Figure 12: Images used in Cardiganise instructions (Phase 2 version), hosted on the reknitrevolution.org website.

Figure 13: Chart guiding calculations for a change in gauge of knitted fabric, part of the replace cuff instructions (Phase 2 version), hosted on the reknitrevolution.org website.

Figure 14: The extended taxonomy of support required for reknitting (Phase 2 version). 